Pacific Journal of Mathematics

ON SATURATED REDUCED PRODUCTS 


\title{
ON SATURATED REDUCED PRODUCTS
}

\author{
Miroslav Benda
}

\begin{abstract}
The first part of this paper characterizes the filters whose reduced products are saturated with respect to quantifier free formulas. It is shown that filters with this property are exactly good filters whose Boolean algebra is compact. In the second part we investigate their set-theoretical properties and prove that such filters exist.
\end{abstract}

This paper contains a proof of the result announced in [1] as well as a number of results directly connected with this theorem.

The problem is to characterize those filters which have the property that products reduced by these filters are $\kappa$-saturated. The special case of the problem in which we talk about ultrafilters only was completely solved by Keisler in [6]. The above problem was first attacked by F. Galvin and also by $\mathrm{Ph}$. Olin and B. Jónsson (see [5]). They showed that the filter of cofinite subsets over $\omega$ is $\omega_{1}$-saturative. Galvin's results were a little more general. In [11], L. Pacholski and C. Ryll-Nardzewski described those atomless filters which were $\omega_{1-}$ saturative. The author of this article then obtained a characterization of $\left(\Phi_{0}, \kappa\right)$-saturative filters for any $\kappa$. S. Shelah after reading a sketch of this article, obtained, using a different method (see [3]), a characterization of $\kappa$-saturative filters, thus solving the problem. (For $\kappa=$ $\omega$, it was independently solved also by L. Pacholski.)

The first part of this paper deals with $\left(\Phi_{0}, \kappa\right)$-saturatedness of reduced products. It is believed that theorems proved here have some applications to algebra. In possible applications the notion $\left(\Phi_{0}, \kappa\right)$-saturatedness seems, better suited than the full saturatedness ([10]) because e.g., a solution of a system of equations is expressible by quantifierfree formulas. In the second part we deal with products of different kind of filters and we apply these results to get an existence theorem for excellent filters. The third part is devoted to discussion and open problems.

Our notation is standard. $\lambda, \kappa$ stand always for cardinals. 0 is the empty set as well as the least element in Boolean algebras. $S_{\omega}(X)$ is the set of finite subsets of $X$. If $f$ is a function from $X$ into $Y$ we write it sometimes as $f: X \rightarrow Y$. If $Z \cong X$ then $f^{*}(Z)=\{f(z) \mid z \epsilon$ $Z$ \} and $f \mid Z$ is the function $f$ restricted to $Z$. A subset $X$ of a Boolean algebra is said to have the finite intersection property if for any $x_{1}, \cdots, x_{n} \in X x_{1} \cap \cdots \cap x_{n} \neq 0$. We write it often as $F I P(X) . \quad D$ usually stands for a filter over the set $I=\cup D$. The ideal $\{x \leqq I \mid I-x \in D\}$ 
is denoted by $J . D$ is called $\sigma$-incomplete if there is a sequence $\left\{X_{n} \mid n<\omega\right\} \subseteq D$ such that $\bigcap_{n>\omega} X_{n}=0 . \quad D$ is $\kappa$-good if it is $\sigma$-incomplete and if for every $f: S_{\omega}(\lambda) \rightarrow D, f$ decreasing (i.e., if $s \subseteq s^{\prime}$ then $f\left(s^{\prime}\right) \leqq f(s)$ ) there is a $g: S_{\omega}(\lambda) \rightarrow D, g$ multiplicative (i.e., $g\left(s \cup s^{\prime}\right)=$ $g(s) \cap g\left(s^{\prime}\right)$ ) and $g(s) \subseteq f(s)$ for each $s \in S_{\omega}(\lambda)$. If $D$ is a filter over $I$ we can form the Boolean algebra $S(I) / D$. Its elements are the sets $[x]_{D}=\{y \mid x-y \in J\} . \quad[x]_{D}$ is often written as $[x] . \quad L$ is a fixed language. If $c$ is a set of constants then $L(c)$ is the language $L$ expanded by $c$. Structures are denoted by Gothic letters and their universes by correspoding Latin capitals. Thus the universe of $\mathfrak{A}_{2}$ is $A_{2}$. If $\phi$ is a formula of $L$ then an instance of $\varphi$ is any formula obtained from $\varphi$ by replacing some of its free variables by constants from $L$. If $\Phi \leqq L$ and $c$ is a set of constants then $\Phi(c)$ is the set of all instances of the formulas from $\Phi$ in the language $L(c)$. If $\Phi \subseteq L$ we say that a structure $\mathfrak{U}$ is $(\Phi, \kappa)$-saturated if for any $c$ and any set of formulas $\Sigma \subseteq \Phi(c)$ such that $|\Sigma|<\kappa$, if $\varphi \in \Sigma$ then $\phi$ has only $v$ as a free variable and

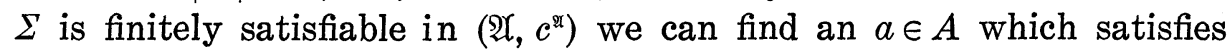
all the formulas in $\Sigma$. Of course $\left(L, \kappa^{+}\right)$-saturatedness coincide with the usual $\kappa^{+}$-saturatedness. The reduced product of the sequence $\left\langle\mathfrak{N}_{i} \mid i \in I\right\rangle$ of structures of the same similarity type (only such products we consider) is denoted by $\Pi \mathfrak{N}_{i} / D . \Phi_{0}$ is the set of quantifierfree formulas of $L$.

1. In this section we define some notions and prove a couple of lemmas which will be useful later.

Definition. A Boolean algebra $\mathscr{B}$ is said to be $\kappa$-compact if for any $X \subseteq B$ such that $F I P(x)$ and $|X|<\kappa$ there is a $b \in B, b \neq 0$ and $b \leqq x$ for any $x \in X$.

LEMma 1. Let $\mathscr{B}$ be a $\kappa$-compact Boolean algebra, let $\lambda<\kappa$ and let $\left\{x_{\alpha} \mid \alpha<\lambda\right\} \subseteq B-\{0\}$ be a set of atomless elements. Then there is $\left\{y_{\alpha} \mid \alpha<\lambda\right\} \leqq B-\{0\}$ such that $y_{\alpha} \leqq x_{\alpha}$ and $y_{\alpha} \cap y_{\beta}=0$ for any $\alpha, \beta<$ $\lambda, \alpha \neq \beta$.

Proof. Using Zorn's lemma we find sets $Z_{\xi}$ for $\xi<\delta$ such that:

(i) $Z_{\xi} \subseteq\left\{x_{\alpha} \mid \alpha<\lambda\right\}$

(ii) $Z_{\xi}$ is a maximal family $Z$ such that $F I P(Z)$ and $Z \cong\left\{x_{\alpha} \mid \alpha<\right.$ $\lambda\}-\mathbf{U}_{\zeta>\xi} Z_{\zeta}$

(iii) $\bigcup_{\xi<\delta} Z_{\xi}=\left\{x_{\alpha} \mid \alpha<\lambda\right\}$.

By $\kappa$-compactness, for each $\xi<\delta$ there is a $c_{\xi} \neq 0$ such that $c_{\xi} \leqq x$ for each $x \in Z_{\xi^{\prime}}$. Clearly, (by (ii)) $c_{\xi} \cap c_{\xi^{\prime}}=0$ if $\xi \neq \xi^{\prime}$. Moreover $c_{\xi}^{\prime} s$ are atomless. So it suffices to show that if $x \in B$ is an atomless element, $x \neq 0$ and $\lambda<\kappa$ then there is $\left\{z_{\alpha} \mid \alpha<\lambda\right\} \subseteq B-\{0\}$ such that 
$z_{\alpha} \leqq x$ and $z_{\alpha} \cap z_{\beta}=0$ for each $\alpha, \beta<\lambda, \alpha \neq \beta$. To show this we define, by induction, the sequence $z^{\prime}$ :

Let $z_{0}^{\prime}=x$. Let us assume that $z^{\prime}$ has been defined for $\xi<\beta$. If $\beta=\beta^{\prime}+1$ let $z_{\beta}^{\prime}$ be a nonzero element which is strictly smaller than $z_{\beta}^{\prime}$, If $\beta$ is limit then by $\kappa$-compactness of $\mathscr{B}$ and by atomlessness of $x$ we can find $z_{\beta}^{\prime}$ so that $z_{\beta}^{\prime} \neq 0$ and $z_{\beta}^{\prime}<z_{\xi}^{\prime}$ for $\xi<\beta$. Having the sequence $z$ we define $z_{\alpha}$ to be $z_{\alpha}^{\prime}-z_{\alpha+1}^{\prime}$ for $\alpha<\lambda$. Evidently, the sequence $\left\{z_{\alpha} \mid \alpha<\lambda\right\}$ has the desired properties.

Lemma 2. Let $r: X \rightarrow S_{\omega}(X)$ and let $R$ be a function on $S_{\omega}(X)$ with the properties that $R(s) \neq 0$ and $R(s) \subseteq \Pi_{x \in s} r(x)$ for every $s \in S_{\omega}(X)$. $I f$, in addition, $R$ satisfies the condition (*),

(*) whenever $s \subseteq s^{\prime}$ and $f \in R\left(s^{\prime}\right)$ then $f \mid s \in R(s)$

then there is an $f \in \Pi_{x \in X} r(x)$ such that $f \mid s \in R(s)$ for each $s \in S_{\omega}(X)$.

Proof. We consider $\Pi_{x \in X} r(x)$ as a usual topogical product of discreet spaces $r(x)$. It is a compact space by Tychonov's theorem. Let $F_{s}=\left\{f \in \Pi_{x \in X} r(x)|f| s \in R(s)\right\} . \quad F_{s}$ is closed for each $s \in S_{\omega}(X)$. The set $\left\{F_{s} \mid s \in S_{\omega}\{X)\right\}$ has the finite intersection property as follows from $\left(^{*}\right)$, so $\bigcap_{s \in S_{\omega}(x)} F_{s} \neq 0$. Any $f \in \bigcap_{s} F_{s}$ will satisfy the lemma.

Definition. A structure $\mathscr{T}=\langle T$, $\leqq>$ is called an upper lattice if $\leqq$ is a partial ordering of $T$ and the lowest upper bound of $\{x, y\}$ exists for any $x, y \in T$; we denote it by $x \vee y$.

A filter $D$ is $\mathscr{T}$-good if it is $\sigma$-incomplete and for any $f: T \rightarrow D$ which is $\leqq$-decreasing (i.e. if $x \leqq y$ then $f(y) \leqq f(x)$ ) there is a $g: T \rightarrow D$ such that $g(x \vee y)=g(x) \cap g(y)$ and $g(x) \subseteq f(x)$ for every $x, y \in T$.

A filter $D$ is said to be $\kappa$-separable if for any $\lambda<\kappa$ and for any $\left\{x_{\alpha} \mid \alpha<\lambda\right\} \subseteq S(I)-J$ such that $x_{\alpha} \cap x_{\beta} \in J$ whenever $\alpha \neq \beta$ there is $\left\{y_{\alpha} \mid \alpha<\lambda\right\} \subseteq S(I)-J$ such that $y_{\alpha} \leqq x_{\alpha}$ and $y_{\alpha} \cap y_{\beta}=0$ for any $\alpha, \beta<$ $\lambda, \alpha \neq \beta$.

REMARKs. (1) A filter is $\kappa$-good in the usual meaning if it is $<S_{\omega}(\lambda)$,

(2) Every filter is $\omega_{1}$-separable.

The following lemma can be proved analogously to Theorem 3.2 in [7]. Just replace $\subseteq$ by $\leqq$ in that proof.

Lemma 3. If $D$ is $\kappa$-good and $|T| \leqq \kappa$ then $D$ is $<T$, $\leqq>-$ good.

Lemma 4. If $D$ is $\kappa$-good filter then $D$ is $\kappa$-separable.

Proof. Let $\left\{x_{\alpha} \mid \alpha<\lambda\right\} \subseteq S(I)-J$ has the property $x_{\alpha} \cap x_{\beta} \in J$ if 
$\alpha \neq \beta$.

Let

$$
f(s)=I-\cup\left\{x_{\alpha} \cap x_{\beta} \mid \alpha, \beta \in s \text { and } \alpha \neq \beta\right\}
$$

for $s \in S_{\omega}(\lambda)$. Then $f: S_{\omega}(\lambda) \rightarrow D$ so, by $\kappa$-goodness, there is a $g: S_{\omega}(\lambda) \rightarrow$ $D$ which is multiplicative and $g(s) \subseteq f(s)$ for $s \in S_{\omega}(\lambda)$. Let $y_{\alpha}=$ $x_{\alpha} \cap g(\{\alpha\})$ for $\alpha<\lambda$. It is clear that $y_{\alpha} \notin J$ for $\alpha<\lambda$. Now, if $\alpha \neq \beta$ then

$$
y_{\alpha} \cap y_{\beta}=x_{\alpha} \cap x_{\beta} \cap g(\{\alpha\}) \cap g(\{\beta\})=x_{\alpha} \cap x_{\beta} \cap g(\{\alpha, \beta\}) .
$$

Since $g(\{\alpha, \beta\}) \subseteq(\{\alpha, \beta\})=I-x_{\alpha} \cap x_{\beta}$ we see that $y_{\alpha} \cap y_{\beta}=0$.

Definition. A set $K \subseteq S(X)$ is called $\kappa$-compact if for any $Z \subseteq$ $K,|Z|<\kappa$ and $F I P(Z)$ we have $\cap Z \neq 0$. If $K \subseteq S(X)$ then $C l(K)$ will be the smallest set $L \subseteq S(X)$ such that $K \cong L$ and whenever $x, y \in L$ then $x \cap y$ and $x \cap y$ belong to $L$ (i.e., $L$ is closed under $U$ and $\cap$ ). The following lemma is well known so the proof of it is omitted.

Lemma 5. Let $K \subseteq S(X) . \quad K$ is $\kappa$-compact iff $C l(K)$ is $\kappa$-compact.

As it was said in the introduction we will be concerned with $(\Phi, \kappa)$-saturatedness of reduced products. It is easily seen from definitions that a structure $\mathfrak{A}$ is $\left(\Phi_{0}, \kappa\right)$-saturated iff the set $\mathscr{D}(\mathfrak{U})$ of all subsets of A which are definable (using parameters) by quantifierfree formulas is $\kappa$-compact. The set $\mathscr{D}(\mathfrak{U})$ is the closure under $U$ and $\cap$ of the set $\mathscr{D}_{0}(\mathfrak{U})$, where $\mathscr{D}_{0}(\mathfrak{U})$ are subsets of $A$ definable by atomic formulas or their negations. Thus, in view of Lemma 5, to show that a structure $\mathfrak{A}$ is $\left(\Phi_{0}, \kappa\right)$-saturated it is enough to show that $\mathscr{D}_{0}(\mathfrak{Z})$ is $\kappa$-compact.

If we analyze the definition of reduced products we see that every member of $\mathscr{D}_{0}\left(\Pi \mathfrak{A}_{i} / D\right)$ can be expressed in one of the following two forms:

(1) $\left\{f / D \mid\left\{i \mid f(i) \in B_{i}\right\} \notin J\right\}$ where $B_{i} \subseteq A_{i}$ for $i \in I$

(2) $\left\{f|D|\left\{i \mid f(i) \in C_{i}\right\} \in J\right\}$ where $C_{i} \subseteq A_{i}$ for $i \in I$.

(Sets of the form (1) are defined by formulas $R\left(v_{0}, \cdots\right)$, sets of the second kind by formulas $\left.\rightarrow R\left(v_{0}, \cdots\right)\right)$. Since sets of the form (1) ((2)) are determined completely by the sequence $B=\left\{B_{i} \mid i \in I\right\}(C=$ $\left.\left\{C_{i} \mid i \in I\right\}\right)$ we will refer to them as $Q_{B}\left(R_{C}\right)$, i.e., $Q_{B}=\{f / D \mid\{i \mid f(i) \in$ $\left.\left.B_{i}\right\} \notin J\right\}$.

2. Definition. A filter $D$ over $I$ is called $\kappa$-excellent if it is $\kappa$-good and if the Boolean algebra $S(I) / D$ is $\kappa$-compact. 
The question of existence of such filters will be discussed in a latter section. We are now ready to prove the main theorem.

THEOREM 1. A filter $D$ is k-excellent iff for every sequence $\left\langle\mathfrak{A}_{i} \mid i \in I\right\rangle$ of structures (of the same type) the reduced product $\Pi \mathfrak{N}_{i} / D$ is $\left(\Phi_{0}, \kappa\right)$-saturated.

Proof. Let us assume that $D$ is a $\kappa$-excellent filter over $I$ and $\left\langle\mathfrak{A}_{i} \mid i \in I\right\rangle$ is a sequence of structures. Let $\lambda<\kappa$ and let $B_{i}^{\alpha}, C_{i}^{\alpha} \subseteq A_{i}$ for every $i \in I$ and $\alpha<\lambda$. Moreover we assume that the set $K=$ $\left\{Q_{B^{\alpha}} \mid \alpha<\lambda\right\} \cup\left\{R_{C^{\alpha}} \mid \alpha<\lambda\right\}$ has finite intersection property. It follows from the discussion of the last section that to prove $\kappa$-saturatedness of $\Pi \mathfrak{N}_{i} / D$ it is enough to show that $\cap K \neq 0$ ( $K$ has been chosen arbitrarily). Let us define

$$
F_{\alpha}(s)=\left\{i \in I \mid B_{i}^{\beta}-\bigcup_{\beta \in s} C_{i}^{\beta} \neq 0\right\}
$$

for $s \in S_{\omega}(\lambda)$ and $\alpha<\lambda$. Because $F I P(K)$ we get that $F_{\alpha}(s) \notin J$ for every $s \in S_{\omega}(\lambda)$ and $\alpha<\lambda$. Note that $F_{\alpha}$ is an increasing function for every $\alpha<\lambda$. Let $\mathscr{B}=S(I) / D$ and let $Y_{\alpha}$ be the set of all atoms of $\mathscr{B}$ which are contained in $\left[F_{\alpha}(s)\right]$ for every $s \in S_{\omega}(\lambda)$ (if $X \subseteq I$ then $[X]$ denoted the corresponding element in the Boolean algebra $S(I) / D)$. We now define $X_{0}, X_{1}, X_{2} \subseteq \lambda$ as follows:

$\alpha \in X_{0}$ iff $\left|Y_{\alpha}\right|<\omega$ and $\left[F_{\alpha}(s)\right]=\cup Y_{\alpha}$ for some $s \in S_{\omega}(\lambda)$

$\alpha \in X_{1}$ iff $\left|Y_{\alpha}\right| \geqq \kappa$

$\alpha \in X_{2}$ iff there is an atomless $b \neq 0$ such that $b \leqq\left[F_{\alpha}(s)\right]$ for every $s \in S_{\omega}(\lambda)$ and $a \notin X_{0} \cup X_{1}$.

We want to show that $X_{0} \cup X_{1} \cup X_{2}=\lambda$. Let $\alpha \in \lambda-\left(X_{0} \cup X_{1}\right)$ and let $\left\{a_{\xi} \mid \xi<\nu\right\}$ be an enumeration of $Y_{\alpha}$. If $\nu<\omega$ then $\left\{\left[F_{\alpha}(s)\right]-\right.$ $\left.\bigcup_{\xi<\nu} a_{\xi} \mid s \in S_{\omega}(\lambda)\right\}$ has the finite intersection property hence we can find a $b \neq 0, b \leqq\left[F_{\alpha}(s)\right]-\bigcup_{\xi<\nu} a_{\xi}$ for every $s \in S_{\omega}(\lambda)$. Since $b$ must be atomless we get that $\alpha \in X_{2}$. Since $\alpha \notin X_{1}$ we must have $\omega \leqq \nu<\kappa$. But then $\left\{\left[F_{\alpha}(s)\right]-a_{\xi} \mid \xi<\nu, s \in S_{\omega}(\lambda)\right\}$ has the finite intersection property and similarly as above we get $\alpha \in X_{2}$.

If $\alpha \in X_{0}$ let $t_{\alpha} \in S_{\omega}(\lambda)$ be an element for which $\left[F_{\alpha}\left(t_{\alpha}\right)\right] \leqq\left[F_{\alpha}(s)\right]$ holds for every $s \in S_{\omega}(\lambda)$, and let $\left[\alpha_{0}^{\alpha}\right], \cdots,\left[\alpha_{n_{\alpha}-1}^{\alpha}\right]$ be the list of atoms included in $\left[F_{\alpha}\left(t_{\alpha}\right)\right]$. We can choose $\alpha_{i}^{\alpha}(\subseteq I)$ in such a way that

$$
\left[a_{i}^{\alpha}\right]=\left[a_{j}^{\beta}\right] \text { implies } a_{i}^{\alpha}=a_{j}^{\beta} \text {. }
$$

Because $\left|X_{1}\right| \leqq \lambda<\kappa$ and $\left|Y_{\alpha}\right| \leqq \kappa$ we can find a sequence $\left\langle b_{\alpha}\right| \alpha \in X_{1}>$ of subsets of $I$, satisfying:

$$
\begin{gathered}
{\left[b_{\alpha}\right] \in Y_{\alpha}} \\
{\left[b_{\alpha}\right] \neq\left[b_{\beta}\right] \text { if } \alpha \neq \beta}
\end{gathered}
$$




\section{$\left[b_{\alpha}\right]$ is distinct from $\left[a_{i}^{\beta}\right]$}

for every $\beta \in X_{0}$ and $\mathrm{i}<n_{\beta}$.

If $\alpha \in X_{2}$ we let $c_{\alpha} \subseteq I$ be such that [ $c_{\alpha}$ ] is non-zero and atomless and for which $\left[c_{\alpha}\right] \leqq\left[F_{\alpha}(s)\right]$ holds for every $s \in S_{\omega}(\lambda)$. Using Lemma 1 we can assume that $\left\{\left[c_{\alpha}\right] \mid \alpha \in X_{2}\right\}$ is a set of mutually disjoint (in $\mathscr{B}$ ) elements. This assumption and (1)-(4) enable us to use Lemma 4, which tells us that we could have chosen $a_{i}^{\alpha}, b_{\alpha}, c_{\alpha}$ in such a way that the set $X=\left\{a_{i}^{\alpha} \mid \alpha \in X_{0}\right.$ and $\left.i<n_{\alpha}\right\} \cup\left\{b_{\alpha} \mid \alpha \in X_{1}\right\} \cup\left\{c_{\alpha} \mid \alpha \in X_{2}\right\}$ is a set of mutually disjoint subsets of $I$. Let $\left\{a_{\xi} \mid \xi<\mu\right\}$ be an enumeration (without repetitions) of $X$. Let $Z_{k}=\left\{\xi \mid a_{\xi} \in X_{k}\right\}$ for $k<3$. Clearly $Z_{i} \cap Z_{j}=$ 0 if $i \neq j$ and $Z_{0} \cup Z_{1} \cup Z_{2}=\mu$.

For any $\alpha \in X_{0}$ we can find an $r \in S_{\omega}\left(Z_{0}\right)$ so that $\left\{F_{\alpha}\left(t_{\alpha}\right)\right]=\mathbf{U}_{\xi \in r}\left[a_{\xi}\right]$. We denote such an $r$ by $r(\alpha)$. If $s \in S_{\omega}\left(X_{0}\right)$ and $t \in S_{\omega}(\lambda)$ we let $P(s, t)$ to be the set of all $f \in \Pi A_{i}$ such that:

(i) $\left\{i \mid f(i) \in B_{i}^{\alpha}\right\} \notin J$ for $\alpha \in s$

(ii) $\left\{i \mid f(i) \in C_{i}^{\alpha}\right\} \in J$ for $\alpha \in t \cup \bigcup_{\beta \in s} t_{\beta}$.

Because $F I P(K)$ holds we have $P(s, t) \neq 0$ for $s \in S_{\omega}\left(X_{0}\right), t \in S_{\omega}(\lambda)$. If $f \in P(s, t)$ and $\alpha \in s$ let $\bar{f}(\alpha)=\beta$ iff $\beta$ is the first $\xi$ for which $\{i \mid f(i) \epsilon$ $\left.B_{i}^{\alpha}\right\} \cap a_{\xi} \notin J$. It follows from the way we defined $t_{\alpha}$ 's that $\bar{f} \in \Pi_{\alpha \in s} r(\alpha)$. Let $R(s, t)=\{\bar{f} \mid f \in P(s, t)\}$. If $t \subseteq t^{\prime}$ then $P\left(s, t^{\prime}\right) \subseteq P(s, t)$ so $R\left(s, t^{\prime}\right) \subseteq$ $R(s, t)$. Thus $R(s)=\bigcap_{t \in S_{\omega}(\lambda)} R(s, t)$ is nonempty since $\Pi_{\alpha \in s} r(\alpha)$ is a finite set. The function $R$ satisfies the condition $\left({ }^{*}\right)$ of Lemma 2 (with $X, Y$ replaced by $X_{0}, Z_{0}$ resp.). Namely, if $s \leqq s^{\prime}$ and $f \in R\left(s^{\prime}\right)$ then for any $t \in S_{\omega}(\lambda)$ there is a $g \in P\left(s^{\prime}, t\right)$ such that $\bar{g}=f$; but such a $g$ is a member of $P(s, t)$ as well as $f|s=\bar{g}| s \in R(s, t)$. Lemma 2 gives us a function $g \in \Pi_{\alpha \in X_{0}} r(\alpha)$ with the property that $g \mid s \in R(s)$ for $s \in$ $S_{\omega}\left(X_{0}\right)$. Let $U_{\alpha}=g^{-1}(\alpha)$ and let $h_{\alpha}$ be a mapping from $\lambda$ onto $U_{\alpha}$. We may assume without loss of generality that $U_{\alpha} \neq 0$ for $\alpha \in Z_{0}$. For $s, t \in S_{\omega}(\lambda)$ and $\alpha \in Z_{0}$ we define:

$$
H_{\alpha}(s, t)=a_{\alpha} \cap\left\{i \mid \bigcap_{\xi \in s} B_{i}^{h_{\alpha}(\xi)}-\bigcup_{\beta \in t} C_{i}^{\beta} \neq 0\right\} .
$$

We want to show that $H_{\alpha}(s, t) \notin J$. Firstly, if $u=\left\{h_{\alpha}(\xi) \mid \xi \in U_{\alpha}\right\}$ then $g^{*}(u)=\{\alpha\}$. Since $g \mid u \in R(u) \subseteq R\left(u, t \cup \cup_{\alpha \in u} t_{\alpha}\right)$ there is an $f \in P(u, t \cup$ $\left.\bigcup_{\alpha \in u} t_{\alpha}\right)$ such that $\bar{f}=g \mid u$. Hence $\left\{i \mid f(i) \in B_{i}^{h} \alpha^{(\xi)}\right\} \cap a_{\alpha} \notin J$ for $\xi \in s$ and $\left\{i \mid f(i) \in C_{i}^{\beta}\right\} \in J$ for each $\beta \in t \cup \bigcup_{\alpha \in u} t_{\alpha}$. The fact that $\left[a_{\alpha}\right]$ is an atom in $\mathscr{B}$ implies that $\left\{i \mid f(i) \in \bigcap_{\xi \in s} B_{i}^{h^{(\xi)}}\right\} \cap a_{\alpha} \notin J$. It is now obvious that $H_{\alpha}(s, t) \notin J$.

If $\alpha \in Z_{1} \cup Z_{2}$ (we let $U_{\alpha}$ be $\{\alpha\}$ and $h_{\alpha}$ be the mapping from $\lambda$ onto $\{\alpha\}$. $H_{\alpha}(s, t)$ for $\alpha \in Z_{1} \cup Z_{2}$ is defined then also by formula (5). Because $h_{\alpha}(\xi)=\alpha$ for $\alpha \in Z_{1} \cup Z_{2}$ we get $H_{\alpha}(s, t) \notin J$ immediately.

Let $a=I-\bigcup_{\alpha<\mu} a_{\alpha}$ and let $\left\{X_{n} \mid n<\omega\right\} \subseteq D$ be such that $\bigcap_{n<\omega} X_{n}=$ 0. Let 


$$
H(r, s, t)=\left(a \cup \bigcup_{\beta \in \mu-r} a_{\beta} \cup \bigcup_{\beta \in r} H_{\beta}(s, t)\right) \cap X_{|r \cup s \cup t|}
$$

for $\left\langle r, s, t>\in T=S_{\omega}(\nu) \times S_{\omega}(\lambda) \times S_{\omega}(\lambda)\right.$. Then $<T$, $>>$, where $\leqq$ is the direct product of the three inclusions, is an upper lattice, so, by Lemma $3, D$ is $\langle T$, $\leqq>$-good. Because $H$ maps $T$ into $D$ and $H$ is $\leqq$-decreasing there is a $G: T \rightarrow D$ such that $G(r, s, t) \subseteq H(r, s, t)$ and $G\left(r \cup r^{\prime}, s \cup s^{\prime}, t \cup t^{\prime}\right)=G(r, s, t) \cap G\left(r^{\prime}, s^{\prime}, t^{\prime}\right)$. Let

$$
d_{\alpha}=a_{\alpha} \cap G(\{a\},\{a\},\{a\}) .
$$

Then

$$
\begin{aligned}
G(\{a\}, s, t) \cap d_{\alpha} & =a_{\alpha} \cap G(\{a\}, s \cup\{\alpha\}, t \cup\{a\}) \\
& \cong a_{\alpha} \cap H(\{\alpha\}, s \cup\{\alpha\}, t \cup\{\alpha\}) \\
& =a_{\alpha} \cap\left(a \cup \bigcup_{\beta \in \mu-\{\alpha\}} a_{\beta} \cup H_{\alpha}(s \cup\{\alpha\}, t \cup\{\alpha\})\right. \\
& =G_{\alpha}(s \cup\{\alpha\}, t \cup\{\alpha\}) \subseteq H_{\alpha}(s, t) .
\end{aligned}
$$

Thus

$$
G(\{\alpha\}, s, t) \cap d_{\alpha} \subseteq H_{\alpha}(s, t) .
$$

We have that $d_{\alpha} \cap d_{\beta}=0$ if $\alpha \neq \beta$ so if $i \in \mathbf{U}_{\alpha<\mu} d_{\alpha}$ we let $\alpha_{i}$ to be that $\alpha$ for which $i \in d_{\alpha}$. Let $T(i)=\left\{\langle\alpha, \beta\rangle \in \lambda \times \lambda \mid i \in G\left(\left\{\alpha_{i}\right\},\{\alpha\},\{\beta\}\right\}\right.$ for $i \in \bigcup_{\alpha} d_{\alpha^{*}} .|T(i)|<\omega$ because $G(r, s, t) \subseteq X_{|r \cup s \cup t|}$ and $i \in G\left(\left\{\alpha_{i}\right\}, V_{i}, W_{i}\right)$ where $V_{i}=\operatorname{Dom} T(i)$ and $W_{i}=R g T(i)$ (this is due to multiplicativity of $G$ ). The last statement is true for every $i \in \mathbf{U}_{\alpha} d_{\alpha}$. It follows from (6) that $i \in H_{\alpha_{i}}\left(V_{i}, W_{i}\right)$. A function $f^{\prime}$ then can be defined on $U_{\alpha} d_{\alpha}$ satisfying

$$
f^{\prime}(i) \in \bigcap_{\xi \in V_{i}} B_{i}^{h \alpha_{i}(\xi)}-\bigcup_{\beta \in W_{i}} C_{i}^{\beta} .
$$

$f^{\prime}$ is a piece of a function $f$ which will belong to $\bigcap_{\alpha} Q_{\beta} \cap \bigcap_{\alpha} R_{C^{\alpha}}$. We check that this piece has the right properties. Let $\gamma<\lambda$. Hence there are $\alpha$ and $\beta$ such that $r=h_{\alpha}(\beta)$. Then

$$
\{i \mid(\exists t)[i \in G(\{\alpha\},\{\beta\}, t)]\} \cap d_{\alpha} \subseteq\left\{i \mid f^{\prime}(i) \in B_{i}^{h}{ }^{(\beta)}\right\}
$$

and

$$
\left\{i \mid f^{\prime}(i) \in C_{i}^{\beta}\right\} \cap \bigcup_{\alpha} d_{\alpha} \subseteq I-\bigcup_{s} \bigcup_{\alpha<\mu} G(\{\alpha\}, s,\{\beta\}) .
$$

Because the left hand side of (7) does not belong to $J$ it is clear that no matter how we extend $f^{\prime}$ to a function from $\Pi A_{i}$ the result will belong to $\bigcap_{\alpha} Q_{\beta \alpha}$. The right side of (8) belongs to $J$. Hence if we extend $f^{\prime}$ to $f$ in such a way that $\left\{i \in I-\bigcup_{\alpha} d_{\alpha} \mid f(i) \epsilon\right.$ $\left.C_{i}^{\beta}\right\} \in J$ for $\beta<\lambda$ we are done $(J$ is closed under $U)$. To do this we define

$$
F(s)=\left(I-\left\{i \mid \mathbf{U}_{\alpha \epsilon s} C_{i}^{\alpha}=A_{i}\right\}\right) \cap X_{|s|}
$$


for $s \in S_{\omega}(\lambda)$. Because $F I P(K)$ we have that $F: S_{\omega}(\lambda) \rightarrow D$. By $\kappa$-goodness of $D$ there is a multiplicative function $G: S_{\omega}(\lambda) \rightarrow D$ such that $G(s) \subseteq F(s)$ for each $s \in S_{\omega}(\lambda)$. Let $t(i)=\{\alpha \mid i \in(\{\alpha\})\}$. Then $i \in G(t(i)) \subseteq$ $F(t(i))$ hence we can define $f^{\prime \prime}$ on $I-\bigcup_{\alpha} d_{\alpha}$ such that $f^{\prime \prime}(i) \in A_{i}$ $\mathrm{U}_{\alpha \in t(i)} C_{i}^{\alpha}$. Consequently, $\left\{i \notin \mathrm{U}_{\alpha} d_{\alpha} \mid f^{\prime \prime}(i) \in C_{i}^{\alpha}\right\} \subseteq I-G(\{\alpha\}) \in J$ for every $\alpha<\lambda$. As we have explained before $f=f^{\prime} \cup f^{\prime \prime}$ is a function beloging to $\bigcap_{\alpha} Q_{B^{\alpha}} \cap \bigcap_{\alpha} R_{C^{\alpha}}$.

For the other direction we assume that every direct product reduced by $D$ is $\left(\Phi_{0}, \kappa\right)$-saturated. In particular $2^{I} / D$ (2 being the 2-element Boolean algebra) is $\left(\Phi_{0}, \kappa\right)$-saturated. Realizing that $2^{I} / D=S(I) / D$ and that $\left(\Phi_{0}, \kappa\right)$-saturatedness of a Boolean algebra is a stronger property than $\kappa$-compactness we get that $S(I) / D$ is $\kappa$-compact.

To prove that $D$ must be $\kappa$-good we take a $\lambda<\kappa$ and a function $f: S_{\omega}(\lambda) \rightarrow D, f$ decreasing. Let $A_{i}=\{s \mid i \in f(s)\}$ and let $\mathfrak{A}_{i}=\left\langle A_{i}\right.$, $>$. If $s \in S_{\omega}(\lambda)$ then $f(s) \in D$ so there is a function $\bar{s} \in \Pi A_{i}$ such that $\{i \mid \bar{s}(i)=s\} \in D$. Let $\Sigma=\left\{c_{s} \subseteq v_{0} \mid s \in S_{\omega}(\lambda)\right\}$. This is a set of quantifier free formulas of the language $L(c)$. Interpreting the constant $c_{s}$ in $\Pi \mathfrak{N}_{i} / D$ by $\bar{s} / D$ we get that $\Sigma$ is finitely satisfiable in $\Pi \mathfrak{N}_{i} / D$; using our assumption we can find an $h \in \Pi A_{i}$ which satisfies $\Sigma$, i.e., $\{i \mid \bar{s}(i) \subseteq$ $h(i)\} \in D$ for each $s \in S_{\omega}(\lambda)$. Let $g(s)=\{i \mid s \leqq h(i)\}$. It is easily seen that $g: S_{\omega}(\lambda) \rightarrow D g(s) \subseteq f(s)$ for $s \in S_{\omega}(\lambda)$ and $g$ is multiplicative.

To prove $\sigma$-incompleteness of $D$ we let $P_{n}=\{m<\omega \mid m \geqq n\}$ for, $n<\omega$ and $\mathfrak{U}_{\xi}=<\omega, P_{n}>_{n<\omega}$. Then $\Sigma=\left\{P_{n}\left(v_{0}\right) \mid n<\omega\right\} \subseteq \Phi_{0}$ is finitely satisfiable in $\mathfrak{A}^{I} / D$, hence there is an $f: I \rightarrow \omega$ such that $\left\{i \mid f(i) \in P_{n}\right\} \in D$ for every $n<\omega$. If $X_{n}=\left\{i \mid f(i) \in P_{n}\right\}$ we have $\left\{X_{n} \mid n<\omega\right\} \subseteq D$ and $\bigcap_{n<\omega} X_{n}=0$. The proof of the theorem is thus completed.

3. Definition. A structure $\mathfrak{A}$ is called rich if for any formula $\varphi$ of $L(\mathfrak{U})$ there is a relational symbol $R$ of $L(\mathfrak{U})$ such that $\mathfrak{A} \Vdash \varphi \leftrightarrow R$. A theory $T$ in a language $L$ is called open if for any formula $\varphi$ of $L$ there is a quantifier-free formula $\psi$ such that $T \vdash \phi \leftrightarrow \psi$.

The proof of the following result can be found in [11].

THEOREM 2. If $\left\{\mathfrak{A}_{i} \mid i \in I\right\}$ is a set of rich structures, $D$ is a filter over $I$ and $S(I) / D$ is atomless then the theory of $\Pi \mathfrak{N}_{i} / D$ is open.

Using this result we get the following corollary to Theorem 1 .

CoRollary 1. If $D$ is $\kappa$-excellent and $\mathscr{B}=S(I) / D$ is atomless then $\Pi \mathfrak{N}_{i} / D$ is $\kappa$-saturated for any sequence $\left\langle\mathfrak{N}_{i} \mid i \in I\right\rangle$.

Proof. We expand $\mathfrak{N}_{i}$ to a rich structure $\mathfrak{N}_{i}^{\prime}$. By Theorem 2 the theory of $\Pi \mathfrak{Y}_{i}^{\prime} / D$ is open. By Theorem $1 \Pi \mathfrak{X}_{i}^{\prime} / D$ is $\left(\Phi_{0}, \kappa\right)$-saturated hence $\Pi \mathfrak{A}_{i}^{\prime} / D$ is $\kappa$-saturated and so is its reduction $\Pi \hat{\vartheta}_{i} / D$. 
We can get a better result than Corollary 1 but to that we need a few more facts.

Lemma 6 (i). If $a \subseteq I, a \notin J$ and $D$ is a $\kappa$-good filter over $I$ then $D(a)$-the filter generated by $D$ and $\{a\}$-is $\kappa$-good.

(ii) If $a \cong I$ is such that $[a]$ is an atom in $S(I) / D$ then $D(a)$ is an ultrafilter.

(iii) If $a \in S(I)-J$ and $S(I) / D$ is $\kappa$-compact then $S(I) / D(a)$ is $\kappa$ compact.

(iv) If $a \in S(I)-J$ and $[a]$ is an atomless element in $S(I) / D$ then $S(I) / D(\alpha)$ is atomless.

Proof. (i ) is almost obvious. (ii), (iii) and (iv) follows from the fact that $S(I) / D(a) \cong\{[x] \mid[x] \leqq[a]\}$, where the last set is viewed as a subalgebra of $S(I) / D$. The isomorphism is given by $[b]_{D(a)} \rightarrow[b \cap a]_{D}$.

The proof of the next theorem involves different method than those discussed here. For a reference, see e.g., [3].

THEOREM 3. If $n<\omega$ and for every $j<n$ the structure $\mathfrak{A}_{j}$ is $\kappa$ saturated then $\Pi_{j<n} \mathfrak{N}_{i}$ is $\kappa$-saturated.

Corollary 2. If $D$ is $\kappa$-excellent and $S(I) / D$ has finitely many atoms then $\Pi \mathfrak{N}_{i} / D$ is $\kappa$-saturated.

Proof. Let $a_{0}, \cdots, a_{n-1} \subseteq I$ be mutually disjoint and such that $\left\{\left[a_{i}\right] \mid i<n\right\}$ is the set of all atoms of $S(I) / D$. Let $a_{n}=I-\bigcup_{i<n} a_{i}$ and let $D_{i}=D\left(a_{i}\right)$ for $i \leqq n$. If follows from Lemma 6(i) and (ii) that for $i<n D_{i}$ is a $\kappa$-good ultrafilter. Lemma 6(i), (iii) and (iv) imply that $D_{n}$ is $\kappa$-excellent and that the algebra $S(I) / D_{n}$ is atomless. By Theorem 2.1 of [6] we get that $\Pi \mathfrak{N}_{i} / D_{j}$ is $\kappa$-saturated for $j<n$. That $\Pi \mathfrak{2}_{i} / D_{n}$ is $\kappa$-saturated we get using Corollary 1 . Note that $D=$ $\bigcap_{j \leqq n} D_{j}$. Using Theorem 1.2 in [4] we get

$$
(\cdot) \Pi \mathfrak{N}_{i} / D \cong \Pi_{j \leqq n}\left(\Pi \mathfrak{W}_{i} / D_{j}\right) \cdot
$$

Theorem 3 yields that the right hand side of $(\cdot)$ is $\kappa$-saturated and so is $\Pi \mathfrak{N}_{i} / D$.

Our next corollary deals with Boolean algebras:

COROLlary 3. If a Boolean algebra $\mathscr{B}$ has at most finitely many atoms and it is of the form $S(I) / D$ where $D$ is a $\kappa$-good filter then $\mathscr{B}$ is $\kappa$-saturated iff $\mathscr{B}$ is $\kappa$-compact.

Proof. It follows from Corollary 2. 
4. Let us pass now to a brief investigation of $\kappa$-excellent filters. We will be primarily interested with products of filters but we will also touch upon questions which will naturally arise.

Throughout this section $D_{k}$ will be a filter over $I_{k}$, where $k \leqq 1$. As before we define $J_{k}=\left\{x \subseteq I_{k} \mid I_{k}-x \in D_{k}\right\}$. If $x \subseteq I_{0} \times I_{1}$ and $i \in I$, then $x^{i}=\left\{j \in I_{0} \mid<j, i>\right\} \in x$. The product $D_{0} \times D_{1}$ of $D_{0}$ and $D_{1}$ is the following filter over $I_{0} \times I_{1}$ :

$$
\left\{x \subseteq I_{0} \times I_{1} \mid\left\{i \mid x^{i} \in D_{1}\right\} \in D_{2}\right\}
$$

The following proposition is obvious.

Proposition 1. $\mathrm{D}_{0} \times D_{1}$ is $\sigma$-incomplete iff $D_{0}$ or $D_{1}$ is $\sigma$-incomplete.

The next result is known as well and is proved in [8].

Proposition 2. $D_{0} \times D_{1}$ is $\kappa$-good iff $D_{1}$ is.

Definition. A filter $D$ is called $\kappa$-compact if the algebra $S(I) / D$ is $\kappa$-compact.

Proposition 3. If $D_{0} \times D_{1}$ is $\kappa$-compact then $D_{1}$ is $\kappa$-compact.

Proof. Let $\left\{x_{\alpha} \mid \alpha<\lambda\right\} \subseteq S\left(I_{1}\right)-J_{1}$ be such that $\left\{\left[x_{\alpha}\right] \mid \alpha<\lambda\right\}$ has the finite interesection property. Let $y_{\alpha}=I_{0} \times x_{\alpha}$. Then $y_{\alpha} \notin J_{0} \times J_{1}$ and $\left\{\left[y_{\alpha}\right] \mid \alpha<\lambda\right\}$ has f.i.p. in $S\left(I_{0} \times I_{1}\right) / D_{0} \times D_{1}$. Let $y \leqq I_{0} \times I_{1}$ be a set satisfying $y \notin J_{0} \times J_{1}$ and $[y] \leqq\left[y_{\alpha}\right]$ for every $\alpha<\lambda$. Then $x=$ $\left\{i \in I_{0} \mid y^{i} \notin J_{0}\right\} \notin J_{1}$ hence $[x] \neq 0$ and it is clear that $[x] \leqq\left[x_{\alpha}\right]$ for every $\alpha<\lambda$.

The other implication is false. Take e.g., $I_{0}=\omega, D_{0}=\{\omega\}, I_{1}=2$ and $D_{1}=\{1\}$. Then $S(2) / D_{1}$ is finite hence saturated, but $S\left(I_{0} \times I_{1}\right) / D_{0} \times$ $D_{1}=S\left(I_{0} \times I_{1}\right)$ is not $\omega_{1}$-compact. Nevertheless we have a partial reverse.

Proposition 4. If $D_{0}$ is an intersection of finitely many ultrafilters over $I_{0}$ and $D_{1}$ is $\kappa$-compact then $D_{0} \times D_{1}$ is $\kappa$-compact.

Proof. Let $D_{0}$ be an intersect on of $n$ distinct ultrafilters. By Theorem 1.2 in [4] we get

$$
2^{I_{0} \times I_{1}} / D_{0} \times D_{1} \cong\left(2^{n}\right)^{I 1} / D_{1} .
$$

( $2^{n}$ is the direct product of $n$ copies of the algebra 2 ).

The referee has suggested the following continuation of the proof 
which is simpler than our original one. Because $\left(2^{n}\right)^{I_{1}} / D_{1} \cong\left(2^{I_{1}} / D_{1}\right)^{n}$ it suffices to show that if $\mathscr{B}_{0}, \cdots \mathscr{B}_{n-1}$ are $\kappa$-compact Boolean algebras then $\mathscr{B}=\mathscr{B}_{0} \times \cdots \times \mathscr{B}_{n-1}$ is also $\kappa$-compact. Indeed, if $\left\{b_{\alpha} \mid \alpha<\lambda\right\}$ $(\lambda<\kappa)$ has f.i.p. in $\mathscr{B}$ and $b_{\alpha}=\left\langle b_{\alpha 0}, \cdots, b_{\alpha n-1}\right\rangle$ then for some $m<n$ $\left\{b_{\alpha m} \mid \alpha<\lambda\right\}$ has f.i.p. in $\mathscr{B}_{m}$. Let $c_{m}$ be such that $0<c_{m} \leqq b_{\alpha m}$ for $\alpha<\lambda$ and let $c_{k}$ be 0 for $k \neq m$. Then $0<\left\langle c_{0}, \cdots, c_{n-1}\right\rangle \leqq b_{\alpha}$ for every $\alpha<\lambda$.

In order to avoid rigid expressions we adopt the following convention:

Convention. We say that a set $\left\{z_{\alpha} \mid \cdots \alpha \cdots\right\} \subseteq S(X)$ has f.i.p. in $S(X) / Y$ if $\left[z_{\alpha}\right]_{Y} \neq 0$ and $\left\{\left[z_{\alpha}\right]_{Y} \mid \cdots \alpha \cdots\right\}$ has f.i.p. in $S(X) / Y$ where $Y$ is a filter over $X$.

We may now ask: What happens if $D_{0}$ is not an intersection of finitely many ultrafilters and $D_{0} \times D_{1}$ is $\kappa$-compact? We naturally expect that $D_{1}$ has some additional property. This property, called fineness is captured in the following definition.

Definition. A filter $D$ over $I$ is called $\kappa$-fine if it is $\sigma$-incomplete and for every $\lambda<\kappa$ and every function $f: S_{\omega}(\lambda) \rightarrow S(I)-J$ which is $\subseteq$-decreasing there is a $g: S_{\omega}(\lambda) \rightarrow S(I)-J$ which is multiplicative and $g(s) \subseteq f(s)$ for every $s \in S_{\omega}(\lambda)$.

REMARK. It follows from the proof of existence of good ultrafilters (see e.g., [7] or [9]) that any filter generated by at most $\kappa$ sets is $\kappa^{+}$-fine (and it is not $\kappa^{+}$-good). For ultrafilters these notions (good and fine) coincide.

Proposition 5. If $D_{0}$ is not intersection of finitely many ultrafilters, $D_{1}$ is $\lambda$-regular for every $\lambda<\kappa$ and $D_{0} \times D_{1}$ is $\kappa$-compact then $D_{1}$ is $\kappa$-fine.

Proof. Since $D_{0}$ is not an intersection of finitely many ultrafilters we can find $\left\{a_{i} \mid i<\omega\right\} \subseteq S\left(I_{0}\right)-J_{0}$ such that $a_{i} \cap a_{j}=0$ if $i \neq j$. Let $\lambda<\kappa$ and let $f: S_{\omega}(\lambda) \rightarrow S\left(I_{1}\right)-J_{1}$ be a decreasing function. Because $D_{1}$ is $\lambda$-regular we can assume without loss of generality that $\bigcap_{\alpha \in X} f(\{\alpha\})=$ 0 for any infinite $X \cong \lambda$. Let $T(i)=\left\{s \in S_{\omega}(\lambda) \mid i \in f(s)\right\}$, where $i \in I_{1}$. Then for every $i \in I_{1},|T(i)|<\omega$, so let $T(i)$ be $\left\{s_{k}^{i} \mid k<n(i)\right\}$. For $\alpha<\lambda$ we define $x_{\alpha} \subseteq I_{0} \times I_{1}$ by

$$
x_{\alpha}^{i}=\bigcup\left\{a_{k} \mid \alpha \in s_{k}^{i}\right\}, i \in I_{1} .
$$

Now $x_{\alpha} \notin J_{0} \times J_{1}$ because $\left.\{i \mid \exists s \in T(i))[\alpha \in s]\right\} \notin J_{1}$ for any $\alpha<\lambda$. We want to show that 


$$
\left\{i \in I_{1} \mid \bigcap_{\alpha \in s} x_{\alpha}^{i} \notin J_{0}\right\}=f(s) .
$$

That the right hand side of (1) is included in the left hand side is obvious. For the other inclusion, if $\bigcap_{\alpha \in s} x_{\alpha}^{i} \notin J_{0}$ there must be a $k<\omega$ such that $a_{k} \subseteq \bigcap_{\alpha \in s} x_{\alpha}^{i}$. Then $\alpha \in s_{k}^{i}$ for every $\alpha \in s$, hence $s \subseteq s_{k}^{i} \in T(i)$, so $i \in f(s)$ as follows from monotonicity of $f$.

Consequently the set $\left\{x_{\alpha} \mid \alpha<\lambda\right\}$ has f.i.p. in $S\left(I_{0} \times I_{1}\right) / D_{0} \times D_{1}$ (see the convention in the previous proof) and by $\kappa$-compactness of $D_{0} \times D_{1}$ there is an $x \in S\left(I_{0} \times I_{1}\right)-J_{0} \times J_{1}$ such that $[x] \leqq\left[x_{\alpha}\right]$ for each $\alpha<\lambda$. Let $d=\left\{i \in I_{1} \mid x^{i} \notin J_{0}\right\}$ and let $g(\{\alpha\})$ be $\left\{i \in d \mid x^{i}-x_{\alpha}^{i} \in J_{0}\right\}$. We extend $g$ to a function on $S_{\omega}(\lambda) g(s)=\bigcap_{\alpha \in s} g(\{\alpha\})$. The $g$ is a multiplicative function from into $S\left(I_{1}\right)-J_{1}$. We have to show now that $g(s) \subseteq f(s)$ for $s \in S_{\omega}(\lambda)$. Let $i \in g(s)$. Then $x^{i}-x_{\alpha}^{i} \in J_{0}$ for each $\alpha \in s$, so $x^{i}-\bigcap_{\alpha \in s} x_{\alpha}^{i} \in J_{0}$ Since $i \in d, x^{i} \notin J_{0}$ hence there must be a $k<\omega$ such that $a_{k} \subseteq \bigcap_{\alpha \in s} x_{\alpha}^{i}$. That means $\alpha \in s_{k}^{i}$ for every $\alpha \in s$ hence $s \leqq s^{i}$ which gives $i \in f(s)$.

The result has the following interesting corollary.

CoRollary. If $D_{0}$ is not an intersection of finitely many ultrafilters, $D_{1}$ is $\lambda$-regular ultrafilter for every $\lambda<\kappa$ and $D_{0} \times D_{1}$ is $\kappa$ compact then $D_{1}$ is $\kappa$-good.

As we have seen before compactness of $D_{1}$ is not enough to assure compactness of $D_{0} \times D_{1}$. Proposition 5 suggests that an additional property should be assumed about $D_{1}$; this suggestion is shown to be true in the next result.

Proposition 6. If $D_{1}$ is $\kappa$-compact and $\kappa$-fine then $D_{0} \times D_{1}$ is $\kappa$ compact and $\kappa$-fine.

Proof. Let $\left\{x_{\alpha} \mid \alpha<\lambda\right\} \subseteq S\left(I_{0} \times I_{1}\right)-J_{0} \times J_{1}$ has f.i.p. in $S\left(I_{0} \times\right.$ $\left.\mathrm{s}_{1}\right) / D_{0} \times D_{1}$. Let

$$
f(s)=\left\{i \in I_{1} \mid \bigcap_{\alpha \in s} x_{\alpha}^{i} \notin J_{0}\right\}
$$

for $s \in S_{\omega}(\lambda) . \quad f$ is a $\subseteq$-decreasing function into $S\left(I_{1}\right)-J_{1}$ so by our assumption there is a $g: S_{\omega}(\lambda) \rightarrow S\left(I_{1}\right)-J_{1}$ which is multiplicative and $g(s) \cong f(s)$ for every $s \in S_{\omega}(\lambda)$. By $\kappa$-compactness of $D_{1}$ there is a $y \in S\left(I_{1}\right)-J_{1}$ such that $y-g(s) \in J_{1}$ (i.e., $\left.[y] \leqq[g(s)]\right)$ for each $s \in S_{\omega}(\lambda)$. Put $t(i)$ equal to $\{\alpha \mid i \in g(\{\alpha\})\}$. Because $D_{1}$ is $\sigma$-incomplete we can assume that $t(i)$ is finite. Let $x$ be that subset of $I_{0} \times I_{1}$ for which the following holds: if $i \in y$ then $x^{i}=\bigcap_{\alpha \in t(i)} x_{\alpha}^{i}$ and if $i \in I_{1}-y$ then $x^{i}=0$. It is easy to check that $x \notin J_{0} \times J_{1}$ and $[x] \leqq\left[x_{\alpha}\right]$ for each $\alpha<\lambda$. The fact that $D_{0} \times D_{1}$ is $\kappa$-fine follows just from the assumption that $D_{1}$ is $\kappa$-fine. The proof is similar to the proof of Proposition 
2 so we leave it to the reader.

Proposition 7. If $D$ is $\kappa$-compact and $\kappa$-good then $D$ is $\kappa$-fine.

Proof. Let $f: S_{\omega}(\lambda) \rightarrow S(I)-J$ be $\subseteq$-decreasing. Thus $\left\{f(s) \mid s \in S_{\omega}(\lambda)\right\}$ has f.i.p. in $S(I) / D$, therefore there is a $Y \subseteq I$ such that $Y-f(s) \in J$ for every $\mathrm{s} \in S_{\omega}(\lambda)$ and $Y \notin J$. Let $F(s)=I-(Y-f(s))$. $\quad F$ is a function from $S_{\omega}(\lambda)$ into $D$, so a function $G: S_{\omega}(\lambda) \rightarrow D$ exists which is multiplicative and $G(s) \subseteq F(s)$. Let $g(s)=G(s) \cap Y$ for $s \in S_{\omega}(\lambda)$. Obviously $g: S_{\omega}(\lambda) \rightarrow S(I)-J$ and $g$ is multiplicative. Moreover, for every $s \in S_{\omega}(\lambda) g(s)=G(s) \cap Y \subseteq F(s) \cap Y=(I-(Y-f(s))) \cap Y \subseteq f(s)$. So $D$ is $\kappa$-fine.

Using the last result and Propositions 2, 3 and 6 we immediately get

Proposition 8. $D_{0} \times D_{1}$ is $\kappa$-good and $\kappa$-compact iff $D_{1}$ is. If $D_{1}$ is $\sigma$-incomplete then we also have that $D_{0} \times D_{1}$ is $\kappa$-excellent iff $D_{1}$ is $\kappa$-excellent.

The last result yields a simple proof of existence of $\kappa$-excellent filters. In fact, for any Boolean algebra $\mathscr{B}$ and any $\kappa$ there is a filter $D$ which is $\kappa^{+}$-excellent and $\mathscr{B} \equiv S(I) / D$. The argument, which is due to Keisler, runs as follows: using Ershov's theorem (see [2]) we find a filter $F$ over $\omega$ such that $\mathscr{B} \equiv S(\omega) / F$. Then we get a $\kappa^{+}$-good $\sigma$-incomplete ultrafilter over $I^{\prime}$ (see [11]) $G$ and we form the filter $D=F \times G$. Now $G$ being an ultrafilter is $\lambda$-compact for any $\lambda$. Hence by Proposition $8 D$ is $\kappa^{+}$-excellent. Because $S(I) D \cong 2^{I} / D \cong$ $\left(2^{\omega} / F\right)^{I^{\prime}} / G \equiv 2^{\omega} / F$ we get $S(I) / D \equiv \mathscr{B}$.

5. This section contains a number of remarks relevant to the topics discussed before as well as some open problems.

(a) We have been investigating various properties of filters. It may be interesting to note that these properties can be put into one of the following three groups: (i) properties which have something to do only with elements of the filter: (ii) properties which have something to do with elements of $S(I)-J$; (iii) properties of the Boolean algebra $S(I) / D$. E.g., goodness, regularity and incompleteness are properties of the first kind. To be fine or to be separable are properties of the second kind while compactness is a property of type (iii). Herewith a set of natural questions is connected. Given a class of filters (e.g., those which make reduced products saturated) we can ask whether the class can be characterized by properties listed under, say, (iii). Or 
we can ask whether "to bə $\kappa$-excellent" cannot be reduced to a property of type (i) (we believe it can't).

(b) Let $\Phi$ be the set of formulas which has the property: if $\varphi \in \Phi$ and $D$ is a filter then $\Pi \mathfrak{A}_{i} / D \vDash \varphi\left(f_{0} / D, \cdots, f_{n} / D\right)$ iff $\left\{i \mid \mathfrak{A}_{i} \vDash \varphi\left(f_{0}(i) \cdots\right)\right\} \notin$ $J$. If $R$ is a relational symbol then $\rightarrow R \in \Phi, \Phi$ is closed under $v$ and if $\varphi \in \Phi$ then $(\mathbb{H} v) \varphi \in \Phi$. Similarly as in [1] we can prove that if $D$ is a $\kappa$-fine filter then $\Pi \mathfrak{A}_{i} / D$ is $(\Phi, \kappa)$-saturated. What other modeltheoretic properties products reduced by fine filters have? The same question can be asked for separable filters.

(c) Are there any natural examples of $\kappa$-compact filters? It is proved in [11] that on $\omega$ every countably generated $\sigma$-incomplete filter is $\kappa$-compact. Is the situation similar on larger cardinals? Specifically, if $F_{k}$ is the filter over $S_{\omega}(\lambda)$ generated by the set $\{\{s \mid \alpha \in s\} \mid \alpha<\kappa\}$ is $F_{\kappa} \kappa^{+}$-compact?

(d) While $\omega_{1}$-complete ultrafilters are rather exceptions there are natural and "small" $\omega$-complete filters. Even though they play an important role in set theory their model-theoretical properties have not been studied in detail. It is conceivable that many results about first order properties of reduced products can be extended to $L_{\omega_{1} \omega_{1}}$ properties of products reduced by $\omega_{1}$-complete filters.

(e) Another notion which could be of interest is $(\kappa, \lambda)$-saturatedness. A structure is $(\kappa, \lambda)$-saturated if every set of formulas of power $\kappa$ is satisfiable in it provided the set has the property that every subset of power $<\lambda$ is satisfiable. Almost every result about saturated structures can be reformulated so a question about $(\kappa, \lambda)$-saturated structures. In connection with this one could ask whether there is a good filter $D$ such that $S(I) / D$ is $\left(\kappa, \omega_{1}\right)$-compact but not $(\kappa, \omega)$-compact (i.e., not $\kappa$-compact). The referee has pointed out that no $\omega_{1}$-complete filter makes all structures $\left(\omega_{1}, \omega_{1}\right)$-saturated.

\section{REFERENCES}

1. M. Benda, On saturated reduced products, Notices 5 (1969), 842.

2. Yu. L. Ershov, Decidability of the elementary theory of relatively complemented distributive lattices and the theories of filters (Russian) Algebra i Logika Sem. 3 (1964), 17.

3. S. Feferman and R. L. Vaught, The first order properties of products of algebraic systems, Fund. Math., 47 (1959), 1957.

4. T. Frayne, A. C. Morel and S. C. Scott, Reduced direct products, Fund. Math., 51 (1962), 195.

5. B. Jónsson and Ph. Olin, Almost direct products and saturation, Composition Math., 20 (1968), 125.

6. H. J. Keisler, Ultraproducts and saturated models, Indag. Math., 26 (1964), 178.

7. Good ideals in fields of sets, Annals of Math., 79 (1964), 338.

8. — Ideals with prescribed degree of goodness, Annals of Math., 81 (1965), 112.

9. K. Kunen, Two teoremsh on ultrafilters, Notices, No. 4, 17 (1970), 673. 
10. J. Mycielski and C. Ryll-Nardzewski, Equationally compact algebras, Fund. Math., LXI (1967), 271.

11. L. Pacholaki and C. Ryll-Nardzewski, On countably compact reduced products (to appear in Fund. Math.).

12. S. Shelah, Generalization of theorems on ultraproducts to reduced product (to appear in Bulletin).

Received October 2, 1970. A large portion of this paper was sketched when the author was under an NSF grant held by Professor Keisler to whom we thank for many stimulating discussions.

University of CALifornia, Berkeley 



\title{
PACIFIC JOURNAL OF MATHEMATICS
}

\author{
EDITORS
}

\author{
H. SAMELSON \\ Stanford University \\ Stanford, California 94305 \\ C. R. HOBBY \\ University of Washington \\ Seattle, Washington 98105
}

J. DugundJI

Department of Mathematics

University of Southern California

Los Angeles, California 90007

RICHARD ARENS

University of California

Los Angeles, California 90024

\section{ASSOCIATE EDITORS}
E. F. BeCKenBaCH
B. H. NeUMANN
F. WOLF
K. Yoshida

\section{SUPPORTING INSTITUTIONS}

\author{
UNIVERSITY OF BRITISH COLUMBIA \\ CALIFORNIA INSTITUTE OF TECHNOLOGY \\ UNIVERSITY OF CALIFORNIA \\ MONTANA STATE UNIVERSITY \\ UNIVERSITY OF NEVADA \\ NEW MEXICO STATE UNIVERSITY \\ OREGON STATE UNIVERSITY \\ UNIVERSITY OF OREGON \\ OSAKA UNIVERSITY
}

\author{
UNIVERSITY OF SOUTHERN CALIFORNIA \\ STANFORD UNIVERSITY \\ UNIVERSITY OF TOKYO \\ UNIVERSITY OF UTAH \\ WASHINGTON STATE UNIVERSITY \\ UNIVERSITY OF WASHINGTON \\ AMERICAN MATHEMATICAL SOCIETY \\ NAVAL WEAPONS CENTER
}

The Supporting Institutions listed above contribute to the cost of publication of this Journal, but they are not owners or publishers and have no responsibility for its content or policies.

Mathematical papers intended for publication in the Pacific Journal of Mathematics should be in typed form or offset-reproduced, (not dittoed), double spaced with large margins. Underline Greek letters in red, German in green, and script in blue. The first paragraph or two must be capable of being used separately as a synopsis of the entire paper. The editorial "we" must not be used in the synopsis, and items of the bibliography should not be cited there unless absolutely necessary, in which case they must be identified by author and Journal, rather than by item number. Manuscripts, in duplicate if possible, may be sent to any one of the four editors. Please classify according to the scheme of Math. Rev. Index to Vol. 39. All other communications to the editors should be addressed to the managing editor, Richard Arens, University of California, Los Angeles, California, 90024.

50 reprints are provided free for each article; additional copies may be obtained at cost in multiples of 50 .

The Pacific Journal of Mathematics is published monthly. Effective with Volume 16 the price per volume (3 numbers) is $\$ 8.00$; single issues, $\$ 3.00$. Special price for current issues to individual faculty members of supporting institutions and to individual members of the American Mathematical Society: $\$ 4.00$ per volume; single issues $\$ 1.50$. Back numbers are available.

Subscriptions, orders for back numbers, and changes of address should be sent to Pacific Journal of Mathematics, 103 Highland Boulevard, Berkeley, California, 94708.

PUBLISHED BY PACIFIC JOURNAL OF MATHEMATICS, A NON-PROFIT CORPORATION

Printed at Kokusai Bunken Insatsusha (International Academic Printing Co., Ltd.), 270, 3chome Totsuka-cho, Shinjuku-ku, Tokyo 160, Japan. 


\section{Pacific Journal of Mathematics}

\section{Vol. 39, No. $3 \quad$ July, 1971}

William O'Bannon Alltop, 5-designs in affine spaces ................... 547

B. G. Basmaji, Real-valued characters of metacyclic groups ................. 553

Miroslav Benda, On saturated reduced products....................... 557

J. T. Borrego, Haskell Cohen and Esmond Ernest Devun, Uniquely representable semigroups. II.......................................

George Lee Cain Jr. and Mohammed Zuhair Zaki Nashed, Fixed points and stability for a sum of two operators in locally convex spaces ....................

Donald Richard Chalice, Restrictions of Banach function spaces ...............

Eugene Frank Cornelius, Jr., A generalization of separable groups ..............

Joel L. Cunningham, Primes in products of rings ......................

Robert Alan Morris, On the Brauer group of $Z$.

593

603

615

David Earl Dobbs, Amitsur cohomology of algebraic number rings ...............

Charles F. Dunkl and Donald Edward Ramirez, Fourier-Stieltjes transforms and

weakly almost periodic functionals for compact groups ...................

Hicham Fakhoury, Structures uniformes faibles sur une classe de cônes et

d'ensembles convexes ......................................

Leslie R. Fletcher, A note on C $\theta \theta$-groups.

Humphrey Sek-Ching Fong and Louis Sucheston, On the ratio ergodic theorem for

semi-groups............................................

James Arthur Gerhard, Subdirectly irreducible idempotent semigroups ...........

Thomas Eric Hall, Orthodox semigroups.....................

Marcel Herzog, $C \theta \theta$-groups involving no Suzuki groups ..........

669

687

John Walter Hinrichsen, Concerning web-like continua ..........

691

Frank Norris Huggins, A generalization of a theorem of F. Riesz.

695

Carlos Johnson, Jr., On certain poset and semilattice homomorphisms

703

Alan Leslie Lambert, Strictly cyclic operator algebras ...........

717

Howard Wilson Lambert, Planar surfaces in knot manifolds . . .

727

Robert Allen McCoy, Groups of homeomorphisms of normed linear spaces ....... 735

T. S. Nanjundiah, Refinements of Wallis's estimate and their generalizations ...... 745

Roger David Nussbaum, A geometric approach to the fixed point index .......... 751

John Emanuel de Pillis, Convexity properties of a generalized numerical range .... 767

Donald C. Ramsey, Generating monomials for finite semigroups ....

783

William T. Reid, A disconjugacy criterion for higher order linear vector differential equations...

Roger Allen Wiegand, Modules over universal regular rings...

Kung-Wei Yang, Compact functors in categories of non-archimedean Banach

spaces.

R. Grant Woods, Correction to: "Co-absolutes of remainders of Stone-Čech compactifications".

Ronald Owen Fulp, Correction to: "Tensor and torsion products of

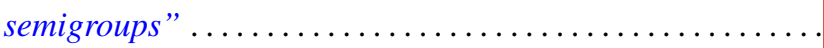

Bruce Alan Barnes, Correction to: "Banach algebras which are ideals in a banach 